\title{
CONTROVERSIES IN THE MANAGEMENT OF ASYMPTOMATIC PATIENTS SUSTAINING PENETRATING THORACOABDOMINAL WOUNDS
}

\author{
Jose Gustavo Parreira, Samir Rasslan, Edivaldo M. Utiyama
}

doi: 10.1590/S1807-59322008000500020

\begin{abstract}
Parreira JG, Rasslan S, Utiyama E. Controversies in the management of asymptomatic patients sustaining penetrating thoracoabdominal wounds. Clinics. 2008;63(5):695-700.

The most challenging diagnostic issue in the management of thoracoabdominal wounds concerns the assessment of asymptomatic patients. In almost one-third of such cases, diaphragmatic injuries are present even in the absence of any clear clinical signs. The sensitivity of noninvasive diagnostic tests is very low in this situation, and acceptable methods for diagnosis are limited to videolaparoscopy or videothoracoscopy. However, these procedures are performed under general anesthesia and present real, and potentially unnecessary, risks for the patient. On the other hand, diaphragmatic hernias, which can result from unsutured diaphragmatic lesions, are associated with considerable morbidity and mortality. In this paper, the management of asymptomatic patients sustaining wounds to the lower chest is discussed, with a focus on the diagnosis of diaphragmatic injuries and the necessity of suturing them.
\end{abstract}

KEYWORDS: Diaphragmatic injury; Videolaparoscopy; Videothoracoscopy; Thoracic trauma; Diaphragmatic hernia.

\section{INTRODUCTION}

The "thoracoabdominal zone" is defined as the area superiorly delimited by the fourth intercostal space (anterior), sixth intercostal space (lateral), and eighth intercostal space (posterior), and inferiorly delimited by the costal margin. ${ }^{1}$ This area is similar to that referred to as the "intrathoracic abdomen," which is the torso region demarcated by the nipple line, costal margins, and scapulae. ${ }^{2}$ The abdominal viscera (i.e., liver and spleen) lie below this area, with their precise location varying based on the movement of the diaphragm muscle, which follows the respiratory cycle. Hence, a penetrating wound in this area could injure the diaphragm muscle or any thoracic viscera (i.e., lung, heart, great vessels, and esophagus), in addition to the abdominal organs. These multiple possibilities make initial diagnostic

Division of Clinical Surgery III, Department of Surgery, Hospital das Clínicas, Faculdade de Medicina, Universidade de São Paulo - São Paulo/ SP, Brasil.

Phone: 55118383.8518

Email: jgparreira@uol.com.br.

Received for publication on February 27, 2008

Accepted for publication on June 12, 2008 assessment in such cases challenging. ${ }^{1-4}$

The standard initial approach to hemodynamically unstable patients sustaining thoracoabdominal injuries is surgery (laparotomy or thoracotomy). ${ }^{5}$ Laparotomy is preferred for injuries exhibiting clear signs of peritonitis. ${ }^{4}$ Operative treatment is also used in stable patients for whom the diagnosis of a cardiac, major vascular, or esophageal injury is defined. ${ }^{4,5}$ This is preceded by a comprehensive diagnostic work-up, which depends on the suspected injury and may involve focused assessment sonography for trauma (FAST), computed axial tomography (CT), angiography, and/or endoscopy.

The most challenging diagnostic issue in this situation concerns asymptomatic patients. ${ }^{1,3,6,7}$ Some studies have reported that diaphragmatic injuries can be present in up to $30 \%$ of such cases despite the absence of clinical signs. Until recently, the sensitivity and negative predictive value of the available diagnostic tests have been considered as very low for the diagnosis of penetrating diaphragmatic wounds. ${ }^{5-10}$ These wounds are usually no more than $2 \mathrm{~cm}$ long, and there is no visceral herniation to the thoracic cavity. ${ }^{1}$ Therefore, the most sensitive diagnostic methods are videolaparoscopy or videothoracoscopy. ${ }^{11-15}$ 
These procedures are undertaken under general anesthesia and present real risks for the patient. However, failure to suture a diaphragmatic tear could ultimately lead to a diaphragmatic hernia. The mortality associated with complicated diaphragmatic hernias is believed to be considerable in this setting. ${ }^{2,3,16}$ Considering these points, approaches to wounds in the lower chest may vary among surgeons and institutions.

Recently published data have shed new light on this subject. Experimental studies have demonstrated that the injured diaphragm can heal and, consequently, development of diaphragmatic hernias is not the rule after penetrating injuries to the diaphragm. ${ }^{17,18}$ Furthermore, no diaphragmatic hernia has been reported after nonoperative treatment of penetrating wounds to the liver, in which diaphragm injuries may be inferred in some cases. ${ }^{19}$ The accuracy of the new generation of computerized tomography scans has been shown to be considerably high for detection of diaphragmatic wounds, and mortality following deferred treatment of diaphragmatic hernias may be lower than previously estimated. ${ }^{20-24}$

Some questions in the management of asymptomatic patients sustaining thoracoabdominal wounds remain unanswered:

1. Should these patients be systematically submitted to operative procedures such as videolaparoscopy or videothoracoscopy in order to diagnose a possible diaphragmatic injury?

2. Should the diaphragmatic injury always be sutured?

2. Can these patients be initially observed, undergoing operative procedures only if a diaphragmatic hernia develops?

2. How high are the morbidity and mortality risks for delayed repair of diaphragmatic hernias?

\section{Incidence of diaphragmatic injuries}

The incidence of diaphragmatic injuries depends mainly on the location of the wound and the mechanism of trauma. Hanna et al. ${ }^{25}$ studied 105 patients with diaphragmatic injuries and found that the external wound was located at the thorax, abdomen, or both in $46 \%, 19 \%$, and $35 \%$ of cases, respectively. Madden et al. ${ }^{2}$ reported that $19 \%$ of the patients who underwent exploratory laparotomies due to penetrating wounds to the lower chest and abdomen sustained diaphragmatic injuries.

Among asymptomatic patients with thoracoabdominal wounds who routinely undergo videolaparoscopy, the incidence of diaphragmatic injuries ranges from $7 \%$ to $48 \%{ }^{1}$ Solda et al. ${ }^{1}$ reported diaphragm injuries in $24.6 \%$ of patients with penetrating wounds to the thoracoabdominal area, and several other studies have reported similar incidences of approximately $20 \% .^{11,12,26}$

Murray et al. ${ }^{6}$ observed these lesions in $42 \%$ of patients with penetrating wounds to the left thoracoabdominal region. Leppäniemi and Haapiainen ${ }^{27}$ reported "occult diaphragm injuries" in $17 \%$ of patients with penetrating trauma to the left thoracoabdominal area. The majority of patients sustaining diaphragmatic lesions have additional associated injuries. The incidence of isolated diaphragmatic injuries ranges from 2 to $12 \% .^{28,29}$

\section{Diagnostic workup to assess diaphragmatic injuries}

It is critical to highlight the difference between blunt and penetrating traumas. Victims of blunt diaphragmatic injuries usually have large defects in the muscle, allowing the abdominal viscera to enter the thoracic cavity. ${ }^{30}$ In these cases, chest X-rays are usually informative. However, the size of the tear in penetrating traumas is smaller, and "real" hernias are infrequent. ${ }^{1,3,6}$ Therefore, any exam that bases diagnosis on the presence of herniated viscera into the thorax is not sufficient for penetrating trauma.

A normal physical examination is observed in $20-45 \%$ of patients with diaphragmatic lesions, and radiological findings are minimal in the majority of these cases ${ }^{1}$. Murray at al. ${ }^{6}$ prospectively studied 119 patients with penetrating injuries to the left thoracoabdominal region. Diaphragmatic injuries were identified in $42 \%$ of patients; of these, $31 \%$ did not have abdominal tenderness, $40 \%$ had normal chest $\mathrm{X}$-rays, and $49 \%$ had an associated hemothorax.

Radiological investigation is useful for victims of gunshot wounds to the thoracoabdominal area, as the trajectory of the projectile can be determined. This is not the case for victims of stab wounds. Diagnoses, therefore, should not be based solely on the physical examination. ${ }^{1,8-10}$ Chest $\mathrm{X}$-ray, wound exploration, and pneumoperitoneum have all been tested, ${ }^{1,2,7-10,16}$ but none has demonstrated sufficient accuracy and reliable negative predictive value. Moore et al. ${ }^{31}$ examined the accuracy of diagnostic peritoneal lavages in detecting diaphragmatic lesions. Using 1,000-10,000 red cells $/ \mathrm{mm}^{3}$ as the laparotomy threshold, they reported $90 \%$ accuracy; however, the negative predictive value was $25 \%$.

Until recently, imaging technology has not been routinely used to assess patients sustaining penetrating injuries to the abdomen. Older technology using spiral CT scans has not shown optimal results in detecting abdominal injuries. ${ }^{8,32}$ Recently published data suggest improved results with more advanced CT equipment. Stein et al. ${ }^{20}$ analyzed the role of multidetector-row $\mathrm{CT}$ in the diagnosis of penetrating injuries to the diaphragm. The authors reviewed the admission CT of 803 patients sustaining penetrating torso injury during 
a four-year period. These scans were classified into three categories: positive, negative, and equivocal. Diaphragmatic injuries were detected in 67 patients and excluded in 736 . If $\mathrm{CT}$ was used to exclude diaphragm injury, the sensitivity (94\%), specificity (95.9\%), and accuracy (95.8\%) were consistently high. Unfortunately, this equipment is not yet widely available. Furthermore, diaphragmatic injuries would remain undetected despite the high accuracy of the exam. We believe that more studies are necessary to confirm the role of multidetector CT scans in the diagnosis of diaphragmatic injuries in penetrating trauma.

\section{Natural course of diaphragmatic wounds}

Diaphragmatic hernias develop over three distinct posttrauma periods. During the acute period directly following trauma, the diaphragm is ruptured, which may be overlooked during diagnosis. This is followed by a quiescent period in which there are no symptoms. The third period is associated with symptoms and, eventually, necrosis of the herniated viscera.

The natural history of diaphragm injuries has not been adequately studied in humans. Without treatment, diaphragmatic healing would be impaired by the pressure gradient between the thorax and abdomen, as well as by respiratory movements. ${ }^{2,17}$ This would facilitate the development of a diaphragmatic hernia, especially on the left side. ${ }^{4,17}$ Complications are also possible on the right side despite the supposed "tamponade" protection offered by the liver. Diaphragmatic hernias have been reported on the right side of patients years after thoracic gunshot wounds. ${ }^{33}$

Perlingeiro et al. ${ }^{17}$ studied the course of diaphragmatic injuries in rats. The diaphragmatic tear was produced by a videolaparoscopy throcar under general anesthesia. Animals were observed for six months, and complete diaphragmatic healing was observed in approximately $90 \%$ of the animals, while diaphragmatic hernias were present in $10 \%$.

A similar phenomenon was observed by Gamblin et al. ${ }^{18}$ who studied 48 rats in an experimental model of diaphragmatic injuries produced by a 16-gauge needle puncture or 2.7-mm aortic punch. Animals were euthanized one month or 10 months after the operative procedure. Only one animal in the aortic punch group, euthanized at 10 months, presented a small hepatic herniation through the diaphragm $(1 / 12=8 \%)$. All other animals demonstrated no signs of herniation.

Of course, these studies do not pertain specifically to humans. The size of the diaphragmatic wound is a very important factor that cannot be controlled for in human trauma cases. However, at least in animals, these data show that the diaphragm can heal without hernia development. But the question remains: should every diaphragmatic wound be sutured?

Several authors have reported nonoperative treatment of penetrating liver injuries. ${ }^{19,34,35}$ This was accomplished by $\mathrm{CT}$ assessment, which revealed the injury through the liver parenchyma. Some of these patients also sustained hemothoraces, and it is possible to infer that they might have had concomitant diaphragmatic injuries ${ }^{19}$. Late diagnosis of diaphragmatic hernias has not yet been reported.

Leppäniemi and Haapiainen ${ }^{27}$ analyzed 97 patients with stab wounds located between the nipple line, umbilical level, and posterior axillary lines, but without immediate indication for laparotomies. These patients were randomized into two groups: clinical observation and surgical exploration (laparotomy or videolaparoscopy). In the surgical exploration group ( $\mathrm{n}=47)$, four patients $(9 \%)$ had diaphragmatic injuries. Three of these injuries were isolated, and thus three of 43 patients would have had occult diaphragmatic injuries if surgical exploration had not been performed. In the observation group $(n=50)$, two patients $(4 \%)$ showed delayed presentation of missed left-side diaphragmatic injuries. However, we cannot definitively state the number of patients with diaphragmatic injuries or the percentage of healing in the group not submitted to operative procedures, as the failure of imaging tests to detect diaphragmatic hernias does not necessarily indicate their absence.

Thus, some diaphragmatic tears on the right side could have been treated without operating, at least in this setting. It is important to stress that there is currently no specific protocol for nonoperative treatment of diaphragmatic injuries. A patient with a diagnosed diaphragmatic injury must currently undergo surgery for repair.

\section{Complications associated with delayed diagnosis}

Potential problem associated with leaving a diaphragmatic wound unsutured concern the reported high morbidity and mortality rates of diaphragmatic hernias when treated in the presence of complications such as bowel necrosis. Feliciano et al. ${ }^{16}$ stated that few topics in trauma surgery have raised more controversy than the delayed diagnosis of a diaphragmatic injury. The consequences are usually not immediate and are often underestimated.

Madden et al. ${ }^{2}$ reviewed selected cases of delayed presentation of diaphragmatic injuries due to stab wounds. The sample included 28 patients, with a mean age of 32 years, and the mortality rate was $36 \%$. Six of the seven patients $(85 \%)$ shown to have gangrenous viscera died. Based on these numbers, an invasive diagnostic workup is justified to eliminate even the smallest risk of overlooking a diaphragmatic injury. 
The reported mortality rates due to diaphragmatic hernias are not consistent, and may not be as high as some authors estimate. Reber et al. ${ }^{22}$ reported the treatment of 10 patients with late presentations of traumatic diaphragmatic hernias. Eight patients sustained blunt trauma, and two were victims of penetrating injuries. Hernias were present on the left side and right side in eight and two patients, respectively. The time elapsed between trauma and clinical presentation ranged from 20 days to 28 years. All of these patients underwent laparotomies, and concomitant thoracotomies were necessary in two cases. Only one patient died (10\% mortality), and three (30\%) sustained postoperative complications.

Bergeron et al. ${ }^{21}$ evaluated the deferred treatment of traumatic diaphragmatic hernias due to blunt trauma. Their study assessed 160 patients sustaining diaphragmatic injuries who were diagnosed immediately after the trauma. Patients undergoing operations for isolated diaphragmatic injuries did not show higher mortality rates due to delayed repair than those undergoing immediate surgery. The authors concluded that blunt diaphragmatic rupture in the absence of other surgical injuries is associated with low mortality, and treatment can be deferred if necessary.

These studies do not specifically address the mortality associated with complicated diaphragmatic hernias (i.e., gangrenous viscera in the thorax). Furthermore, some of these studies assessed victims of blunt trauma for whom the diaphragm defect was expected to be larger and the risk of viscera strangulation was lower. ${ }^{21,22}$ Recently, several studies have reported low mortality in the treatment of complicated diaphragmatic hernias. ${ }^{16,23,24,36-38}$ Nevertheless, the rate of complications and mortality in the treatment of complicated diaphragmatic hernias needs to be further addressed.

\section{The active search for penetrating diaphragmatic wounds}

The ideal treatment should be based on the preoperative diagnosis of the diaphragmatic injury, such that only patients sustaining these injuries undergo operation. However, current imaging technology limits proper diagnosis. Considering the possible complications associated with unsutured diaphragmatic wounds, a protocol for actively searching for diaphragmatic injuries is justified. Currently, the most sensitive and specific methods are videolaparoscopy and videothoracoscopy. ${ }^{1,11-15}$

The advantages of videosurgery are evident: the diaphragm can be directly viewed and easily assessed, ${ }^{1,12-15}$ muscular stretching permits better visualization of the defect, and concomitant abdominal lesions may be observed and treated. Moreover, the contents of the thoracic cavity can be aspirated and the diaphragmatic lesion laparoscopically treated. ${ }^{14,15}$

Concerns related to creating a hypertensive pneumothorax with the pneumoperitoneum has not been shown to be a major problem. ${ }^{39}$ The diaphragmatic lesion can usually be recognized in patients sustaining stab wounds.

The situation is a bit more complicated when gunshot wounds are the cause of trauma, as the trajectory is often unpredictable. Assessment of the splenic flexure of the colon in the left upper quadrant is often difficult. Some caution is required to completely rule out other injuries. Perforations in the small bowel and colon may be difficult to see, especially when hematomas or free blood are present in the abdominal cavity. ${ }^{1,40}$ Finally, visualization of the posterior portion of the diaphragm with videolaparoscopy may be difficult, particularly in posterolateral wounds.

The sensitivity and specificity of videolaparoscopy are very convincing. Friese et al. ${ }^{26}$ prospectively studied 34 hemodynamically stable and asymptomatic patients sustaining penetrating thoracoabdominal wounds. All patients underwent diagnostic laparoscopy to assess the diaphragm, followed by confirmatory celiotomy or videothoracoscopy. There were seven true positive, 30 true negative, one false negative, and no false positive tests. The specificity (100\%), sensitivity (87.5\%), and negative predictive value $(96 \%)$ were all high. The single false negative case was due to a hemoperitoneum associated with a splenic lesion that warranted laparotomy.

Solda et al. ${ }^{1}$ studied asymptomatic patients sustaining penetrating wounds to the thoracoabdominal area. All patients routinely underwent videolaparoscopy. A total of 73 patients, 62 of whom were victims of stab wounds, were analyzed. Diaphragmatic injury was diagnosed in 18 patients (24\%), eight (44\%) of whom demonstrated isolated diaphragmatic injuries. Laparoscopic suture was possible in six of these cases.

Videolaparoscopy allows not only diagnosis, but also treatment in selected cases ${ }^{40,41}$. Zantut et al. ${ }^{41}$ examined the use of videolaparoscopy in penetrating abdominal traumas. Of the 510 patients studied, laparotomy was avoided in 277 (54\%) and diaphragmatic injuries were laparoscopically treated in 16 cases. Only 10 patients presented complications, all of which considered minor.

Videothoracoscopy is another available method used to assess asymptomatic patients with thoracoabdominal wounds. ${ }^{14,15}$ It aids assessment of the posterior portion of the diaphragm. Selective tracheal intubation is not essential, but is recommended. The pneumoperitoneum is not needed, further thoracic evaluation may be carried out (i.e., pericardium window and projectile trajectory), and some lesions can be treated (i.e., lung suture). It is always 
our policy to perform a videolaparoscopy or laparotomy to evaluate the abdomen in cases of diaphragmatic lesion diagnosed by videothoracoscopy.

Dorgan et al. ${ }^{15}$ studied the role of videothoracoscopy in 51 patients sustaining thoracic trauma. Nine of 24 patients $(37.5 \%)$ with thoracoabdominal wounds sustained diaphragmatic injuries. Videothoracoscopy failed to detect a large right-sided diaphragmatic rupture due to blunt trauma. This patient underwent videothoracoscopy 30 days after the trauma, with a diagnosis of retained hemothorax. Seven days after the initial operative procedure, the patient underwent thoracotomy and a $15-\mathrm{cm}$ right-sided diaphragmatic rupture was noticed.

There is some debate concerning whether videolaparoscopy or videothoracoscopy is the best option. Each method has an appropriate indication. In asymptomatic patients with penetrating wounds to the thoracoabdominal area, a videothoracoscopy should be performed in two situations: if placement of a thoracic tube is required (pneumothorax or hemothorax), or cardiac injury is suspected and a pericardial window is to be performed. In all other cases, videolaparoscopy is the best alternative.

\section{Videosurgery assessment or clinical observation for asymptomatic patients sustaining wounds to the thora- coabdominal area}

This question, related to that of suturing diaphragm wounds, unfortunately remains unanswered. For many years, suturing of diaphragm injuries has been the rule. However, there are risks associated with active searches using videosurgery, and a selective protocol may be warranted. Prospective data detailing the long-term results of pure clinical observations of patients sustaining wounds to the thoracoabdominal zone are not currently available.

Leppäniemi \& Haapiainen ${ }^{27}$ studied 97 patients with stab wounds located between the nipple line, umbilical level, and posterior axillary lines. Forty-seven underwent surgical intervention in order to search for diaphragmatic injuries, which were found in four patients (9\%). In the observation group $(n=50)$, two patients $(4 \%)$ presented with left diaphragmatic injuries after two and 23 months. Considering these numbers, we may estimate that around $50 \%$ of patients with left-side diaphragmatic injuries will develop hernias within the first year after the injury.

It is clear that we lack the evidence to formulate a rule for these cases. If the surgeon believes that every diaphragmatic injury should be sutured, the price to be paid is a $70 \%$ rate of negative videolaparoscopies, in addition to the risks of anesthesia, iatrogenic injuries, and costs. If one chooses not to search for diaphragmatic injuries, the unpleasant diagnosis of a delayed diaphragmatic hernia may occur in some cases. The risk of developing a hernia seems to be small, but strangulation rates in patients that do develop hernias can be high. An active search with a multidetector CT during follow-up may be the best course of action for diagnosing diaphragmatic defects before serious complications develop.

Demetriades et al. ${ }^{35}$ described an algorithm for treating hemodynamically stable patients sustaining thoracoabdominal wounds without peritoneal signs. In this protocol, videolaparoscopy is indicated if the wound is located on the left thoracoabdominal area. These researchers accepted the risks associated with not suturing right diaphragmatic tears, but active searching for left diaphragmatic lesions was routinely indicated. We accept that asymptomatic patients sustaining penetrating wounds to the right thoracoabdominal zone may eventually be observed in hospitals where nonoperative treatment protocols are implemented. However, our current opinion is that even right diaphragmatic lesions should be sutured because complicated right-sided diaphragmatic hernias have been observed in clinical practice. ${ }^{33}$ Therefore, we believe that the majority of asymptomatic patients sustaining penetrating wounds to the thoracoabdominal region should undergo obligatory invasive investigation via videolaparoscopy or videothoracoscopy.

\section{FINAL COMMENTS}

In conclusion, diaphragmatic injuries are difficult to diagnose in asymptomatic patients sustaining wounds to the thoracoabdominal area. With the development of multidetector CT scans, preoperative diagnoses may improve, but some diaphragmatic wounds may still be overlooked. Although it is possible for diaphragmatic tears to heal without intervention, overlooking lesions remains a major concern.

The reported incidence of diaphragmatic injuries in patients with wounds to the thoracoabdominal area varies widely, from $7 \%$ to $48 \%$, depending on the methodology of the study. It seems that the chance of developing a hernia is small; however, if a hernia does develop, strangulation of the herniated viscera becomes a serious risk. More studies assessing the mortality of complicated diaphragmatic hernias are needed, as insufficient data are currently available to definitively state a rule in these cases. Although some surgeons accept the practice of not suturing a right-sided diaphragmatic tear, active searching for a tear on the left side of the diaphragm is warranted. Videolaparoscopy and videothoracoscopy are reliable methods for ruling out these injuries, especially if the wound is located on the left thoracoabdominal area. 


\section{REFERENCES}

1. Solda S, Rasslan S, Rodrigues FCM. Videolaparoscopia diagnóstica nos ferimentos da transição tóraco-abdominal. Rev Col Bras Cir. 1996;30711.

2. Madden MR, Paull DE, Finkelstein JL, Goodwin CW, Marzulli V, Yurt RW et al. Occult diaphragmatic injuries from stab wounds to the lower chest and abdomen. J Trauma. 1989;29:292-8.

3. Demetriades D, Kakoyiannis S, Parekh D, Hatzitheofilou C. Penetrating injuries of the diaphragm. Br J Surg. 1988;75:824-6.

4. Gianninni JA, Rasslan S, Silva LE, Coimbra R, Saad Júnior R. Ferimentos penetrantes tóraco-abdominais e de tórax e abdome: análise comparativa da morbidade e mortalidade pós-operatórias. Rev Col Bras Cir. 1998;297-303.

5. Parreira JG, Soldá S, Rasslan S. Análise dos indicadores de hemorragia letal em vítimas de trauma penetrante de tronco admitidas em choque: Um método objetivo para selecionar os candidatos ao "controle de danos". Rev Col Bras Cir. 2002;29:256-66.

6. Murray JA, Demetriades D, Cornwell III EE, Asensio JA, Velmahos G, Belzberg $\mathrm{H}$ et al. Penetrating left thoracoabdominal trauma: The incidence and clinical presentation of diaphragmatic injuries. J Trauma. 1997;43:624-6.

7. Boyle EM, Maier R, Salazar JD, Kovacich JC, O'Keefe G, Mann FA, et al. Diagnosis of injuries after stab wounds to the back and flank. J Trauma. 1997;42:260-5.

8. Ginzburg E, Carrillo E, Kopelman T, McKenney MG, Kirton OC, Shatz $\mathrm{DV}$, et al.The role of computed tomography in selective management of gunshot wounds to the abdomen and flank. J Trauma. 1998;45:10059.

9. Pikoulis E, Delis S, Scandalakis P, Leppäiniemi AK, Derlopas K, Geranios A, et al. Reliability of initial chest radiographs in the diagnosis of blunt diaphragmatic rupture. Annales Chirurgiae et Gynaecologiae. 2000;89:10-3.

10. Merlotti GJ, Dillon B, Lange DA, Robin AP, Barrett J. Peritoneal lavage in penetrating thoracoabdominal trauma. J Trauma. 1988;28:17-23.

11. Powell BS, Magnotti LJ, Schroeppel TJ, Finnell CW, Savage AS, Fischer PE, et al. Diagnostic laparoscopy for the evaluation of occult diaphragmatic injury following penetrating thoracoabdominal trauma. Injury. 2008; Mar 10 [Epub ahead of print].

12. Ivatury RR, Simon RJ, Weksler B, Bayard V, Stahl WM. Laparoscopy in the evaluation of the intrathoracic abdomen after penetrating injury. J Trauma. 1992;33:101-08.

13. Ivatury RR, Simon RJ, Stahl WM. A critical evaluation of laparoscopy in penetrating abdominal trauma. J Trauma. 1993;34:822-8.

14. Jones JW, Kitahame A, Webb WR, McSwain N. Emergency thoracoscopy: a logical approach to chest trauma management. J Trauma. 1981;21:280-3.

15. Dorgan Neto V, Saad Júnior R, Rasslan S. Videotoracoscopia no trauma de tórax. Rev Col Bras Cir. 2001;28:3-8.

16. Feliciano DV, Cruse PA, Mattox KL, Bitondo CG, Burch JM, Noon GP, et al. Delayed diagnosis of injuries of the diaphragm after penetrating wounds. J Trauma. 1988;28:1135-43.

17. Giannini Perlingeiro JA, Saad Junior R, Lancelotti CL, Rasslan S, Candelaria PC, Solda SC. Natural course of penetrating diaphragmatic injury: na experimental study. Int Surg. 2007;92:1-9.

18. Gamblin TC, Wall CE Jr, Morgan JH 3rd, Erickson DJ, Dalton ML, Ashley DW. The natural history of untreated penetrating diaphragm injury: an animal model. J Trauma. 2004;57:989-92.

19. Renz BM, Feliciano DV. Gunshot wounds to the right thoracoabdomen: a prospective study of nonoperative management. J Trauma. 1994;37:73744.

20. Stein DM, York GB, Boswell S, Shanmuganathan K, Haan JM, Scalea TM. Accuracy of computed tomography (CT) scan in the detection of penetrating diaphragm injury. J Trauma. 2007;63:538-43.
21. Bergeron E, Clas D, Ratte S, Beauchamp G, Denis R, Evans D, et al. Impact of deferred treatment of blunt diaphragmatic rupture: a 15-year experience in six trauma centers in Quebec. J Trauma. 2002;52:63340.

22. Reber PU, Schmied B, Seiler CA, Baer HU, Patel AG, Büchler MW. Missed diaphragmatic injuries and their long-term sequelae. J Trauma. 1998;44:183-8.

23. Abboud B, Jaoude JB, Riachi M, Sleilaty G, Tabet G. Intrathoracic transverse colon and small bowel infarction in a patient with traumatic diaphragmatic hernia. Case report and review of the literature. J Med Liban. 2004;52:168-70.

24. Alimoglu O, Eryilmaz R, Sahin M, Ozsoy MS. Delayed traumatic diaphragmatic hernias presenting with strangulation. Hernia. 2004;8:393-6.

25. Hanna WC, Ferri LE, Fata P, Razek T, Mulder DS. The current status of traumatic diaphragmatic injuriesÇ lessons learned from 105 patients over 13 years. Ann Thorac Surg. 2008;85:1044-8.

26. Friese RS, Coln CE, Gentilello LM. Laparoscopy is sufficient to exclude occult diaphragm injury after penetrating abdominal trauma. J Trauma. 2005;58:789-92.

27. Leppäniemi A, Haapiainen R. Occult diaphragmatic injuries caused by stab wounds. J Trauma. 2003;55:646-50.

28. Pagliarelo G, Cartr J. Traumatic injury to the diaphragm: timely diagnosis and treatment. J Trauma. 1992;33:194-7.

29. Borlase BC, Metcalf RK, Moore EE, Manart FD. Penetrating wounds to the anterior chest. Analysis of thoracotomy and laparotomy. Am J Surg. 1986; 152:649-52.

30. Guth AA, Pachter L, Unsup K. Pitfalls in the diagnosis of blunt diaphragmatic injury. Am J Surg. 1995;170:5-9.

31. Moore J, Moore EE, Thompson JS. Abdominal injuries associated with penetrating trauma in the lower chest. Am J Surg. 1980;140:724-9.

32. Rehn CG, Sherman R, Hinz TW. The role of CT scan in the evaluation for laparotomy in patients with stab wounds of the abdomen. J Trauma. 1989;29:446-50.

33. Baldassarre E, Valenti G, Gambino M, Arturi A, Torino G, Porta IP, et al. The role of laparoscopy in the diagnosis and the treatment of missed diaphragmatic hernia after penetrating trauma. J Laparoendosc Adv Surg Tech A. 2007;17:302-6.

34. Renz BM, Bott J, Feliciano DV. Failure of nonoperative treatment of a gunshot wound to the liver predicted by computed tomography. J Trauma. 1996;40:191.

35. Demetriades D, Hadjizacharia P, Constantinou C, Brown C, Inaba $\mathrm{K}$, Rhee $\mathrm{P}$, et al. Selective nonoperative management of penetrating abdominal solid organ injuries. Ann Surg. 2006;40:620-8.

36. Abboud B, Tabet G, Bou Jaoude J, Sleilaty G. Gastric incarceration and perforation following posttraumatic diaphragmatic hernia: case report and review of the literature. J Med Liban. 2007;55:104-7.

37. Makhija R, Akoh JA. Strangulated diaphragmatic hernia presenting clinically as pericarditis. World J Gastroenterol. 2007;13:1449-50.

38. Baca B, Karahasanoğlu T, Saribeyoğlu K, Arica P, Kol E. Late complication of diaphragmatic gunshot injury: appendix perforation due to colon incarceration. Ulus Travma Acil Cerrahi Derg. 2007;13:70-3.

39. Soldá SC, Rodrigues FCR, Rasslan S, Perlingeiro JAG, de Figueiredo LFP, Rocha e Silva M. Repercussões respiratórias e hemodinâmicas do pneumoperitônio na lesão do diafragma - estudo experimental. Rev Assoc Med Bras. 2007;53:294-9.

40. Solda SC, Rodrigues FCM, Martins L, Pinto MCC, Rasslan S. Lesão diafragmática isolada por ferimento penetrante tratada por videolaparoscopia. Rev Col Bras Cir. 1994;21:213-6.

41. Zantut LF, Ivatury RR, Smith RS, Kawarrara NT, Porter JM, Fry WR, et al. Diagnostic and therapeutic laparoscopy for penetrating abdominal trauma: a multicenter experience. J Trauma. 1997;42:825-9. 